\title{
Bootstrapping Matrix Quantum Mechanics
}

\author{
Xizhi Han (韩希之), Sean A. Hartnoll, and Jorrit Kruthoff॰ \\ Department of Physics, Stanford University, Stanford, California 94305-4060, USA
}

(Received 20 May 2020; accepted 2 July 2020; published 22 July 2020)

\begin{abstract}
Large $N$ matrix quantum mechanics is central to holographic duality but not solvable in the most interesting cases. We show that the spectrum and simple expectation values in these theories can be obtained numerically via a "bootstrap" methodology. In this approach, operator expectation values are related by symmetries-such as time translation and $S U(N)$ gauge invariance-and then bounded with certain positivity constraints. We first demonstrate how this method efficiently solves the conventional quantum anharmonic oscillator. We then reproduce the known solution of large $N$ single matrix quantum mechanics. Finally, we present new results on the ground state of large $N$ two matrix quantum mechanics.
\end{abstract}

DOI: 10.1103/PhysRevLett.125.041601

Introduction.-Large $N$ matrices are at the heart of the holographic emergence of semiclassical, gravitating spacetime geometry [1]. In matrix quantum mechanics geometry emerges from an underlying theory with no built in locality. The simplest such theory is the single matrix quantum mechanics description of two-dimensional string theory [2], while the richest are the maximally supersymmetric multimatrix theories of BFSS [3] and BMN [4]. There are many theories in between, with varying numbers of matrices and degrees of supersymmetry [5]. Thus far, only the single matrix quantum mechanics has proved solvable at large $N[6]$.

Nonzero temperature Monte Carlo studies of large $N$ multimatrix quantum mechanical systems have successfully captured aspects of a known dual spacetime in supersymmetric theories [7-10]. Substantial Monte Carlo studies have also been performed for nonzero temperature bosonic multimatrix theories, e.g., Refs. [11,12]. However, recent work increasingly suggests that the quantum structure of holographic quantum states-revealed, for instance, in their entanglement [13-16] - plays a central role in the emergence of space. It therefore behooves us to find methods suitable for studying the zero temperature quantum states of multimatrix quantum mechanics directly. Progress was made recently in this direction by using a neural network variational wave function [17]. Here we describe a different approach.

Our work is directly inspired by a recent beautiful paper by Lin [18], with a similar approach also being employed in Ref. [19]. Lin's paper studied large $N$ matrix integrals,

Published by the American Physical Society under the terms of the Creative Commons Attribution 4.0 International license. Further distribution of this work must maintain attribution to the author(s) and the published article's title, journal citation, and DOI. Funded by SCOAP ${ }^{3}$. which is an easier problem than large $N$ quantum mechanics but shares important features. Positivity constraints and relations between correlation functions were shown to efficiently produce strong numerical bounds on correlation functions of matrix integrals. In the following we will show how this methodology can be adapted to the quantum mechanical problem.

Bootstrapping the quantum anharmonic oscillator.-We first illustrate the approach with a warm-up example of a quantum anharmonic oscillator, with Hamiltonian

$$
H=p^{2}+x^{2}+g x^{4} .
$$

Here $[p, x]=-i$. Figure 1 below shows the results for this case: strong constraints on the energy $E$ and expectation value $\left\langle x^{2}\right\rangle$ of the ground state and first excited state.

The first step is to relate the expectation values of different operators. We will obtain the recursion relation in Eq. (6) below. In energy eigenstates, for any operator $\mathcal{O}$,

$$
\langle[H, \mathcal{O}]\rangle=0 .
$$

For example, let $\mathcal{O}=x p$. Equation (2) is then the Virial theorem, $\left\langle 2 p^{2}\right\rangle=\left\langle 2 x^{2}+4 g x^{4}\right\rangle$. The energy is therefore

$$
E=2\left\langle x^{2}\right\rangle+3 g\left\langle x^{4}\right\rangle .
$$

More systematically, take $\mathcal{O}=x^{s}$ and $\mathcal{O}=x^{t} p$ in Eq. (2) for integers $s, t \geq 0$. Commuting the operators $x$, $p$ with the identity $\left[p, x^{r}\right]=-i r x^{r-1}$ and eliminating the terms with a single $p$ operator, we arrive at the relation

$$
4 t\left\langle x^{t-1} p^{2}\right\rangle=8 g\left\langle x^{t+3}\right\rangle+4\left\langle x^{t+1}\right\rangle-t(t-1)(t-2)\left\langle x^{t-3}\right\rangle .
$$

In this single particle case is there is a strengthened version of Eq. (2): $\langle\mathcal{O} H\rangle=E\langle\mathcal{O}\rangle$. We emphasize Eq. (2) instead because, as we will see later, it is more useful in the 

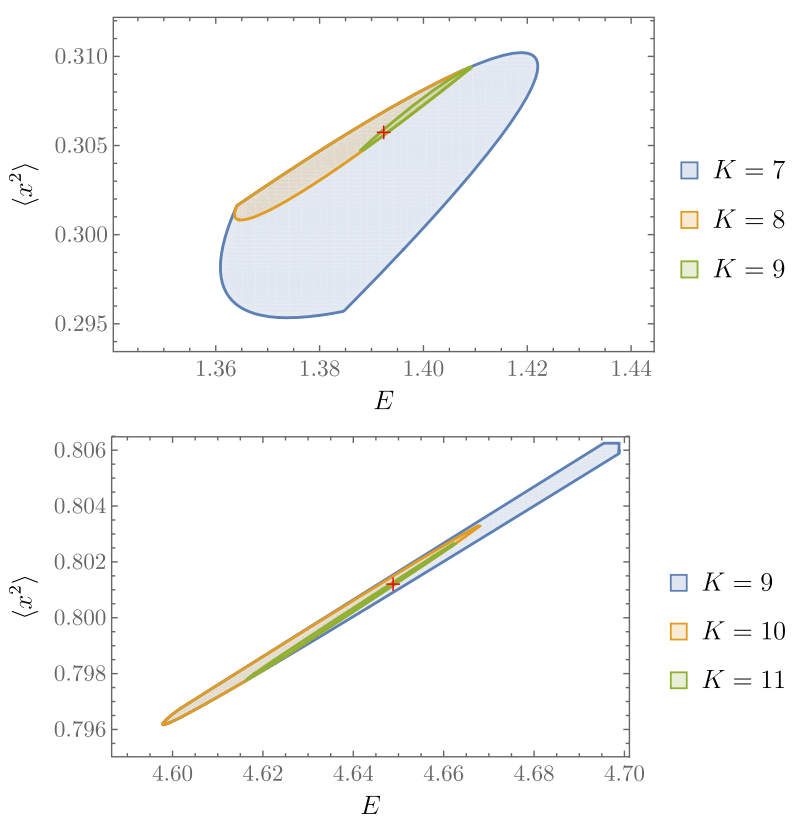

FIG. 1. Bootstrap allowed region (shaded) for the anharmonic oscillator (1) with $g=1$. Upper plot: the allowed region for $\left(E,\left\langle x^{2}\right\rangle\right)$ near the ground state solution (marked by the red cross) for different sizes of the bootstrap matrix $K=7,8$, 9; lower plot: the allowed region near the first excited state.

matrix case. Nonetheless, in the present anharmonic oscillator example, take $\mathcal{O}=x^{t-1}$, so that

$$
\left\langle x^{t-1} p^{2}\right\rangle=E\left\langle x^{t-1}\right\rangle-\left\langle x^{t+1}\right\rangle-g\left\langle x^{t+3}\right\rangle .
$$

Plugging Eq. (5) into Eq. (4) gives a recursive relation between expectation values of powers of $x$ :

$$
\begin{aligned}
& 4 t E\left\langle x^{t-1}\right\rangle+t(t-1)(t-2)\left\langle x^{t-3}\right\rangle \\
& -4(t+1)\left\langle x^{t+1}\right\rangle-4 g(t+2)\left\langle x^{t+3}\right\rangle=0,
\end{aligned}
$$

where $E$ is given by Eq. (3). Also we know that $\left\langle x^{0}\right\rangle=1$ and $\left\langle x^{t}\right\rangle=0$ if $t$ is odd, so all expectation values of $x^{t}$ can be computed from $E$ and $\left\langle x^{2}\right\rangle$ with Eq. (6).

With the recursion relation (6) at hand we move onto the second step. We wish to solve for $E$ and $\left\langle x^{2}\right\rangle$, the only two unknown variables, by bootstrapping. This step works as in Ref. [18]. The basic positivity constraint is that

$$
\left\langle\mathcal{O}^{\dagger} \mathcal{O}\right\rangle \geq 0, \quad \forall \mathcal{O}=\sum_{i=0}^{K} c_{i} x^{i},
$$

which means that the matrix $\mathcal{M}$ of size $(K+1) \times(K+1)$, $\mathcal{M}_{i j}=\left\langle x^{i+j}\right\rangle$, should be positive semidefinite. The constraint becomes stronger as we increase $K$, thus enlarging the space of trial operators. For a given $K$ and test values of $E$ and $\left\langle x^{2}\right\rangle$, the $\mathcal{M}_{i j}$ can be computed using the recursion

relation (6). The bootstrap consists of scanning over these test values, computing the eigenvalues of the matrix $\mathcal{M}$, and thereby determining if positivity excludes the test values as inconsistent.

The result is shown in Fig. 1. Even for moderate $K$ the values of $E$ and $\left\langle x^{2}\right\rangle$ are determined quite accurately. The region of allowed values splits into a discrete set of islands. These converge to the spectrum of the Hamiltonian in the limit $K \rightarrow \infty$ [20]. Higher energy states require more constraints to be computed accurately.

One matrix quantum mechanics.-Now we generalize the bootstrap method to matrix quantum mechanics at $N=\infty$. The momentum operators can no longer be eliminated explicitly in favor of the energy, and we do not use a closed form recursion relation for all expectation values. However, the energy and expectation values of short operators can still be efficiently constrained.

Consider the single-matrix quantum mechanics with

$$
H=\operatorname{tr} P^{2}+\operatorname{tr} X^{2}+\frac{g}{N} \operatorname{tr} X^{4},
$$

where $P$ and $X$ are $N$-by- $N$ Hermitian matrices with quantum commutators $\left[P_{i j}, X_{k l}\right]=-i \delta_{i l} \delta_{j k}$. The theory (8) can be solved by mapping onto $N$ free fermions [6]. The bootstrap reproduces this solution in Fig. 2.

Operator expectation values are related by symmetries. In the following, denote $\langle\mathcal{O}\rangle=\operatorname{tr} \rho \mathcal{O}$. If the state $\rho$ commutes with the Hamiltonian then

$$
\langle[H, \mathcal{O}]\rangle=0, \quad \forall \mathcal{O} .
$$

For example, $\rho$ could be a pure energy eigenstate or a mixed thermal state. Choosing $\mathcal{O}=\operatorname{tr} X P$,

$$
2\left\langle\operatorname{tr} P^{2}\right\rangle=2\left\langle\operatorname{tr} X^{2}\right\rangle+\frac{4 g}{N}\left\langle\operatorname{tr} X^{4}\right\rangle .
$$

The $S U(N)$ symmetry of Eq. (8) has generators

$$
G=i[X, P]+N I .
$$

The final identity piece ensures that $\langle\operatorname{tr} G\rangle=0$, with the operator ordering $[X, P]=X P-P X$ in Eq. (11). In gauged matrix quantum mechanics, physical states must be invariant under this symmetry. In particular,

$$
\langle\operatorname{tr} G \mathcal{O}\rangle=0, \quad \forall \mathcal{O}_{i j} .
$$

For example, $\langle\operatorname{tr} G\rangle=0$ implies $\langle\operatorname{tr} X P\rangle-\langle\operatorname{tr} P X\rangle=i N^{2}$. Combining this constraint with $\left\langle\left[H, \operatorname{tr} X^{2}\right]\right\rangle=0$ gives

$$
\langle\operatorname{tr} X P\rangle=-\langle\operatorname{tr} P X\rangle=\frac{i N^{2}}{2} .
$$

Cyclicity of the trace gives another set of relations between operators. Commuting quantum operators may 
be necessary in applying the cyclic formula. For example, using large $N$ factorization to leading order in $N \rightarrow \infty$,

$$
\left\langle\operatorname{tr} X P^{3}\right\rangle=\left\langle\operatorname{tr} P^{3} X\right\rangle+2 i N\left\langle\operatorname{tr} P^{2}\right\rangle+i\langle\operatorname{tr} P\rangle\langle\operatorname{tr} P\rangle .
$$

Equations (9), (12), cyclicity of the trace, and reality conditions $\left\langle\mathcal{O}^{\dagger}\right\rangle=\langle\mathcal{O}\rangle^{*}$ generate all relations between expectation values that we will use for the bootstrap.

As a mini-bootstrap example, consider trial operators $I$, $X, X^{2}$, and $P$. From the condition (7), the following bootstrap matrix should be positive semidefinite:

\begin{tabular}{c|cccc} 
& $I$ & $X^{2}$ & $X$ & $P$ \\
\hline$I$ & $\langle\operatorname{tr} I\rangle$ & $\left\langle\operatorname{tr} X^{2}\right\rangle$ & 0 & 0 \\
$X^{2}$ & $\left\langle\operatorname{tr} X^{2}\right\rangle$ & $\left\langle\operatorname{tr} X^{4}\right\rangle$ & 0 & 0 \\
$X$ & 0 & 0 & $\left\langle\operatorname{tr} X^{2}\right\rangle$ & $\langle\operatorname{tr} X P\rangle$ \\
$P$ & 0 & 0 & $\langle\operatorname{tr} P X\rangle$ & $\left\langle\operatorname{tr} P^{2}\right\rangle$.
\end{tabular}

Trial operators are built from both $X$ and $P$. The expectation value for an odd number of matrices vanishes. Positivity of Eq. (15) implies

$$
\begin{aligned}
& \left\langle\operatorname{tr} X^{2}\right\rangle \geq 0, \quad N\left\langle\operatorname{tr} X^{4}\right\rangle \geq\left\langle\operatorname{tr} X^{2}\right\rangle^{2}, \\
& \left\langle\operatorname{tr} X^{2}\right\rangle\left(\left\langle\operatorname{tr} X^{2}\right\rangle+\frac{2 g}{N}\left\langle\operatorname{tr} X^{4}\right\rangle\right) \geq \frac{N^{4}}{4},
\end{aligned}
$$

where Eqs. (10) and (13) are used. The inequalities (16) are the bootstrap constraints in this simple example. At $g=0$, $\left\langle\operatorname{tr} X^{2}\right\rangle=\frac{1}{2} N^{2}$ and $\left\langle\operatorname{tr} X^{4}\right\rangle=\frac{1}{2} N^{3}$, so the last inequality in Eq. (16) is saturated and the other two are not.

The bootstrap constraints become stronger as we include more trial operators. First, take all possible strings of $X$ and $P$ of length $\leq L$, and write down the matrix analogous to Eq. (15). This matrix must be positive semidefinite. Second, regard each of the $\sim 2^{2 L}$ entries in the matrix as a variable (which is the expectation value of a single-trace operator with length $\leq 2 L$ ), and write down the equalities between them following from Eqs. (9), (12), cyclicity of the trace, $\left\langle\mathcal{O}^{\dagger}\right\rangle=\langle\mathcal{O}\rangle^{*}$ and that the expectation value of an odd number of matrices vanishes. The technical implementation of these constraints, as well as the minimization described in the following paragraph, is detailed in Ref. [21].

Unlike in the single-particle case, we do not necessarily require that the state be an energy eigenstate and the energy $E$ does not appear explicitly in the bootstrap constraints. At infinite $N$ the matrix quantum mechanics has a continuous spectrum and therefore we proceed to use gradient descent to minimize the energy in the allowed region of expectation values. In this way we obtain a lower bound on the ground state energy of the theory. The result is a lower bound because certainly the true ground state energy is allowed, and hence above the minimal allowed energy that we find. In Fig. 2 we observe that the lower bound is very close to
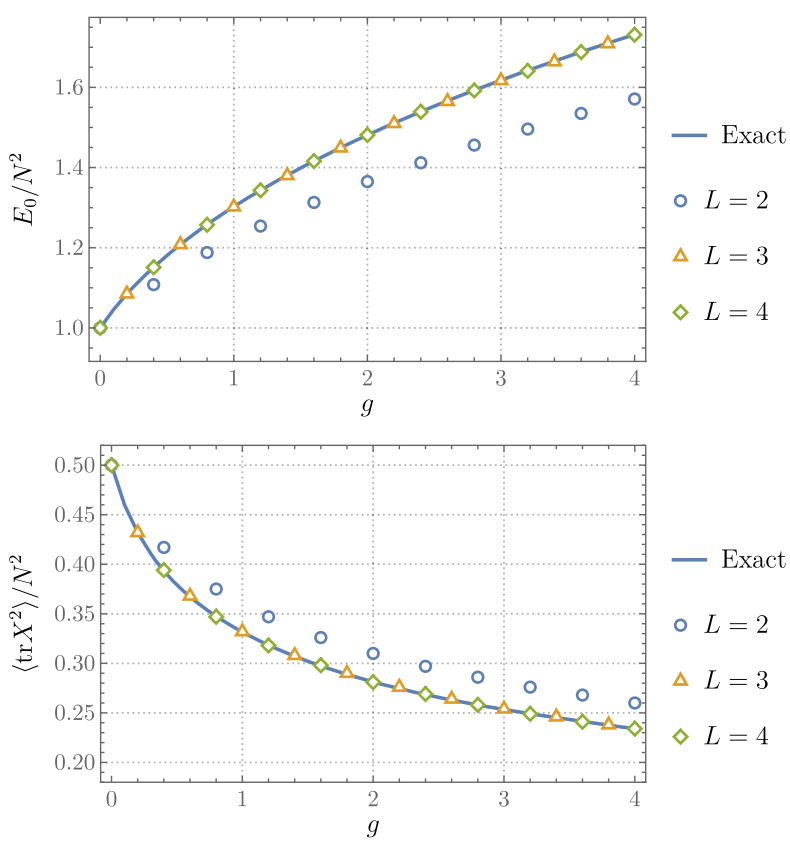

FIG. 2. One matrix quantum mechanics bootstrap for the Hamiltonian (8). $L$ is the maximal length of trial operators. Upper: The markers show the minimal energies allowed by the bootstrap constraints, in comparison with the exact ground state solution. Lower: the expectation values of $\operatorname{tr} X^{2}$, for the minimal energy parameters found in the upper plot.

the true ground state value, already for $L=3$, and other observables, such as $\left\langle\operatorname{tr} X^{2}\right\rangle$, are also solved accurately.

Two matrix quantum mechanics. - One matrix quantum mechanics are tractable analytically as one can diagonalize the matrix. This is not the case for multimatrix quantum mechanics. In the following we illustrate how bootstrap methods can successfully be used for such theories, focusing on a relatively simple two-matrix quantum mechanics with a global $O(2)$ symmetry (in addition to the large $N$ gauge symmetry). The Hamiltonian is

$$
H=\operatorname{tr}\left\{P_{X}^{2}+P_{Y}^{2}+m^{2}\left(X^{2}+Y^{2}\right)-g^{2}[X, Y]^{2}\right\},
$$

with $X$ and $Y$ being $N$-by- $N$ Hermitian matrices, with conjugate momenta $P_{X}$ and $P_{Y}$, and $m$ and $g$ coupling constants. This theory is not exactly solvable. An early discussion of the massless $(m=0)$ limit of the theory is Ref. [25]. By rescaling the matrices we see that dimensionless physical quantities can only depend on the ratio $m^{2} / g^{4 / 3}$.

Imposing rotational invariance gives more relations between observables. We expect the ground state to be rotationally invariant. Rotations are generated by

$$
S=\operatorname{tr}\left(X P_{Y}-Y P_{X}\right)
$$

For states $\rho$ with $[S, \rho]=0$, including eigenstates of $S$, 


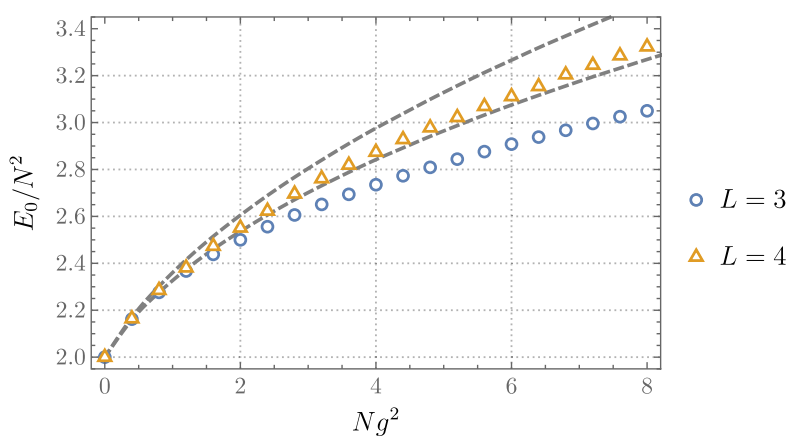

As further evidence that the $L=4$ bootstrap results are close to convergence, we compare our results to existing low temperature Monte Carlo simulations of the massless theory. At large $g, E_{0} / N^{2} \approx 1.40\left(N g^{2}\right)^{1 / 3}+$ $1.01 \mathrm{~m}^{2} /\left(\mathrm{Ng}^{2}\right)^{1 / 3}$ from data in Fig. 3. The factor of 1.40 agrees precisely with the Monte Carlo result in Ref. [26], corresponding to the value of 0.70 in the conventions of that paper. An analogous fit gives the leading order behavior $\left\langle\operatorname{tr} X^{2}+\operatorname{tr} Y^{2}\right\rangle / N^{2} \approx 1.22 /\left(N g^{2}\right)^{1 / 3}$. The numerical factor here is close to the Monte Carlo result of 1.15 in Ref. [26].

The $S U(N)$ gauge invariance allows us to diagonalize one of the two matrices, say $X$. Let the eigenvalues be $x_{i}$. The Hamiltonian for the entries $y_{i j}$ of the remaining matrix is a sum of harmonic oscillators, with frequencies $\omega_{i j}^{2}=m^{2}+g^{2}\left(x_{i}-x_{j}\right)^{2}$. We can therefore write down a Born-Oppenheimer wave function in which these oscillators are placed in their ground state:

$$
\Psi(X, Y)=\psi\left(x_{i}\right) \prod_{i, j=1}^{N}\left(2 \omega_{i j} / \pi\right)^{1 / 4} e^{-\frac{1}{2} \omega_{i j}\left|y_{i j}\right|^{2}} .
$$

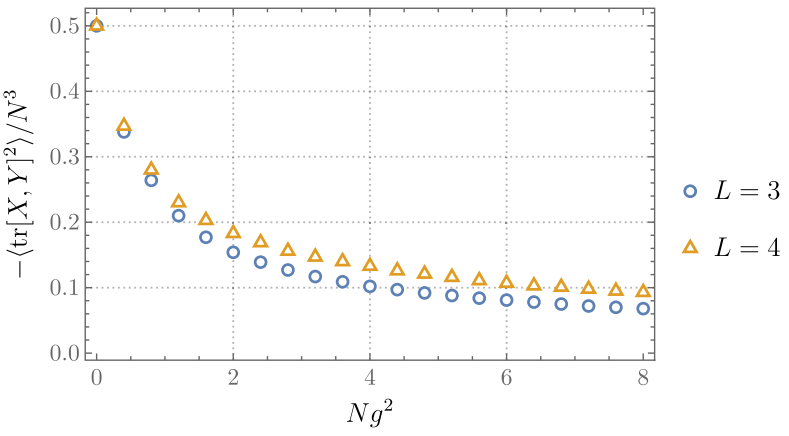

FIG. 3. Minimal energy configuration in the bootstrap allowed region for $L=3,4$. The gray dashed curves are rigorous lower and upper bounds of the ground state energy from the BornOppenheimer approximation. In the plots we have set $m=1$.

$$
\langle[S, \mathcal{O}]\rangle=0, \quad \forall \mathcal{O} \text {. }
$$

Thus in the two matrix quantum mechanics, Eqs. (9), (12), and (19), cyclicity of the trace, and $\left\langle\mathcal{O}^{\dagger}\right\rangle=\langle\mathcal{O}\rangle^{*}$ will be used to generate all equations between expectation values that we will use. The bootstrap then proceeds in exactly the same way as for the case of a single matrix, now with $\sim 4^{2 L}$ variables prior to imposing constraints. The results for the ground state energy, $\left\langle\operatorname{tr} X^{2}+\operatorname{tr} Y^{2}\right\rangle$ and $\left\langle\operatorname{tr}[X, Y]^{2}\right\rangle$ are in Fig. 3. The Virial theorem relates these: $E_{0}=2 m^{2}\left\langle\operatorname{tr} X^{2}+\operatorname{tr} Y^{2}\right\rangle-3 g^{2}\left\langle\operatorname{tr}[X, Y]^{2}\right\rangle$.

In order to corroborate the accuracy of the $L=4$ results, we obtain rigorous upper and lower bounds on the true ground state energy using a Born-Oppenheimer wave function. We see in Fig. 3 that the $L=4$ bootstrap results indeed lie within a narrow window allowed by these bounds. We briefly describe the wave function in the following paragraph, with details given in Ref. [21].

That is, the $y_{i j}$ are treated as "fast" compared to the eigenvalues $x_{i}$. Born-Oppenheimer wave functions lead to both upper and lower bounds on the ground state energy. The upper bound follows from treating the wave function as a variational ansatz. The lower bound is obtained by finding the ground state of the eigenvalues in an effective potential due to the zero point energy of the $y_{i j}$ oscillators. The advantage of the form (20) is that computing the upper and lower bounds reduces to a solvable single-matrix large $N$ eigenvalue problem. In Fig. 3 we see that the bounds following from the wave function (20) turn out to be remarkably tight.

From the results in Fig. 3 one can verify that the ratio $N \operatorname{tr}[X, Y]^{2} /\left(\operatorname{tr} X^{2}\right)^{2}$ tends to a nonzero constant at large $N g^{2}$. This means that the matrices do not commute in this limit. This can be contrasted with the analogous two matrix integral, with no time, that does become commuting at large $N g^{2}$ [27]. This is consistent with the fact that the two matrix integral diverges in the massless limit [28,29], as the eigenvalues spread far apart along the classically flat directions of the potential due to commuting matrices, while the massless matrix quantum mechanics still has a discrete spectrum of normalizable states [30].

Final comments. - In summary, we have introduced a systematic numerical method to obtain energies and expectation values of the large $N$ matrix quantum mechanics states. The method involves establishing relationships between expectation values and then imposing positivity of a certain matrix of expectation values, in the spirit of [18]. In Fig. 2 we see that the known analytic results for one-matrix large $N$ quantum mechanics are readily reproduced. In Fig. 3 we have obtained new results for the 
ground state energy and expectation values of a two matrix large $N$ quantum mechanics.

The extension to more matrices should be possible with increased computing power or perhaps by optimizing the algorithm. Looking at supersymmetric states in supersymmetric theories may allow for stronger relationships between expectation values, using the supersymmetry generators. Both more matrices and supersymmetry will of course be necessary to tackle the full blown theories of Banks, Fischler, Shenker, and Susskind [3] and Berenstein, Maldacena, and Nastase [4]. Finally, extensions to the Gibbs states (or, to high energy eigenstates) may allow nonzero temperature quantum physics to be accessed with our bootstrap methods. This could give an alternative probe of the thermal phase transitions studied via Monte Carlo simulations in, e.g., Refs. [11,12], as well as a new window into black hole microstates.

This work arose from discussions with Edward Mazenc and Daniel Ranard, who also collaborated on the early stages of the project. J. K. is supported by the Simons Foundation. S. A. H. is partially supported by DOE Award No. de-sc0018134 and by a Simons Investigator Grant.

[1] J. M. Maldacena, Int. J. Theor. Phys. 38, 1113 (1999).

[2] I. R. Klebanov, in String Theory and Quantum Gravity '91, Proceedings of the Trieste Spring School and Workshop (World Scientific, 1991), pp. 30-101.

[3] T. Banks, W. Fischler, S. H. Shenker, and L. Susskind, Phys. Rev. D 55, 5112 (1997).

[4] D. E. Berenstein, J. M. Maldacena, and H. S. Nastase, J. High Energy Phys. 04 (2002) 013.

[5] B. de Wit, Nucl. Phys. B, Proc. Suppl. 56B, 76 (1997).

[6] E. Brezin, C. Itzykson, G. Parisi, and J. Zuber, Commun. Math. Phys. 59, 35 (1978).

[7] K. N. Anagnostopoulos, M. Hanada, J. Nishimura, and S. Takeuchi, Phys. Rev. Lett. 100, 021601 (2008).

[8] S. Catterall and T. Wiseman, Phys. Rev. D 78, 041502(R) (2008).

[9] V. G. Filev and D. O'Connor, J. High Energy Phys. 05 (2016) 167.

[10] E. Berkowitz, E. Rinaldi, M. Hanada, G. Ishiki, S. Shimasaki, and P. Vranas, Phys. Rev. D 94, 094501 (2016).
[11] T. Azuma, T. Morita, and S. Takeuchi, Phys. Rev. Lett. 113, 091603 (2014).

[12] G. Bergner, N. Bodendorfer, M. Hanada, E. Rinaldi, A. Schfer, and P. Vranas, J. High Energy Phys. 01 (2020) 053.

[13] E. Bianchi and R. C. Myers, Classical Quantum Gravity 31, 214002 (2014).

[14] T. Faulkner, A. Lewkowycz, and J. Maldacena, J. High Energy Phys. 11 (2013) 074.

[15] W. Donnelly and L. Freidel, J. High Energy Phys. 09 (2016) 102.

[16] D. Harlow, Commun. Math. Phys. 354, 865 (2017).

[17] X. Han and S. A. Hartnoll, Phys. Rev. X 10, 011069 (2020).

[18] H. W. Lin, J. High Energy Phys. 06 (2020) 090.

[19] P. D. Anderson and M. Kruczenski, Nucl. Phys. B921, 702 (2017).

[20] If $\langle I\rangle=1,\left\langle\mathcal{O}^{\dagger}\right\rangle=\langle\mathcal{O}\rangle^{*}$, and $\left\langle\mathcal{O}^{\dagger} \mathcal{O}\right\rangle \geq 0$ for all operators $\mathcal{O}$, then $\langle\mathcal{O}\rangle=\operatorname{tr}(\rho \mathcal{O})$ for some quantum state $\rho$. If, furthermore, $\langle\mathcal{O} H\rangle=E\langle\mathcal{O}\rangle$, then $\rho$ must be an eigenstate with energy $E$. Therefore as $K \rightarrow \infty$, wherein the constraints are indeed imposed for all operators, the allowed region of energies necessarily shrinks to the spectrum of the Hamiltonian, with $\langle\mathcal{O}\rangle$ the expectation value in energy eigenstates.

[21] See Supplemental Material at http://link.aps.org/ supplemental/10.1103/PhysRevLett.125.041601 for details of numerical implementation and of the Born-Oppenheimer wavefunction, which includes Refs. [22-24].

[22] S. R. Das and A. Jevicki, Mod. Phys. Lett. A 05, 1639 (1990).

[23] O. Aharony, J. Marsano, S. Minwalla, K. Papadodimas, M. Van Raamsdonk, and T. Wiseman, J. High Energy Phys. 01 (2006) 140.

[24] J. Nocedal and S. Wright, Numerical Optimization (Springer Science \& Business Media, New York, 2006).

[25] J. Hoppe, Quantum theory of a massless relativistic surface and a two-dimensional bound state problem, Ph.D. thesis, Massachusetts Institute of Technology, 1982.

[26] T. Morita and H. Yoshida, Phys. Rev. D 101, 106010 (2020).

[27] D. E. Berenstein, M. Hanada, and S. A. Hartnoll, J. High Energy Phys. 02 (2009) 010.

[28] W. Krauth, H. Nicolai, and M. Staudacher, Phys. Lett. B 431, 31 (1998).

[29] W. Krauth and M. Staudacher, Phys. Lett. B 435, 350 (1998).

[30] B. Simon, Ann. Phys. (N.Y.) 146, 209 (1983). 